TRANSACTIONS OF THE

AMERICAN MATHEMATICAL SOCIETY

Volume 349, Number 4, April 1997, Pages 1515-1525

S 0002-9947(97)01892-8

\title{
A FOUR-DIMENSIONAL DEFORMATION OF A NUMERICAL GODEAUX SURFACE
}

\author{
CARYN WERNER
}

\begin{abstract}
A numerical Godeaux surface is a surface of general type with invariants $p_{g}=q=0$ and $K^{2}=1$. In this paper the moduli space of a numerical Godeaux surface with order two torsion is computed to be eightdimensional; whether or not the moduli space of such a surface is irreducible is still unknown. The surface in this paper is constructed as one member of a four parameter family of double planes. There is a natural involution on the surface, inherited from the double plane construction, which acts on the moduli space. We show that the invariant subspace is four-dimensional and coincides with the family of double planes.
\end{abstract}

\section{INTRODUCTION}

In [W], a minimal surface $\tilde{X}$ of general type was constructed with the invariants $p_{g}=q=0, K^{2}=1$; we call such a surface a numerical Godeaux surface. In this paper we construct a four-dimensional family of surfaces deforming $\tilde{X}$, and then compute the cohomology of the tangent sheaf $\Theta_{\tilde{X}}$ to show that the moduli space of the surface is locally smooth and eight-dimensional.

This surface has torsion group Tors $\mathrm{H}_{1}(\tilde{X}, \mathbb{Z})=\mathbb{Z} / 2$. Other examples of numerical Godeaux surfaces with order two torsion have been constructed by Barlow ([Ba]) and Inoue ([I]). The irreducibility of the moduli space of numerical Godeaux surfaces with trivial or order two torsion is still unknown, while in each of the remaining cases Tors $=\mathbb{Z} / 3, \mathbb{Z} / 4$, and $\mathbb{Z} / 5$, the moduli space has been shown to be irreducible and eight-dimensional.

The surface $\tilde{X}$ is constructed as the minimal model of a double plane $X$ branched along a degree ten curve. The branch curve has five infinitely near triple points, one ordinary quadruple point, and no other singularities; after blowing up the plane to resolve the singularities, the double cover is formed branched along the resulting smooth curve. This double plane construction of a numerical Godeaux surface was first suggested by Campedelli ([Ca]), and the first exhibited is due to Oort and Peters ([O-P]), whose example has torsion and fundamental group equal to $\mathbb{Z} / 4$.

The branch curve in the original construction of $\tilde{X}$ is the union of an octic and a conic; in $\S 1$, we use a birational transformation of the plane to write the branch curve as the union of a sextic and a quartic; using this configuration, we then construct a four-dimensional family of such curves in $\S 2$. In the last two sections we show that the moduli space of $\tilde{X}$ is eight-dimensional and smooth at $\tilde{X}$, and

Received by the editors September 10, 1995.

1991 Mathematics Subject Classification. Primary 14J29, 14J10.

(C)1997 American Mathematical Society 
the family of double covers is the four-dimensional invariant subspace of the moduli space.

\section{A QUADRATIC TRANSFORMATION OF THE PLANE}

The branch curve constructed in $[\mathrm{W}]$ is an octic with an infinitely near triple point $p_{1}$, a quadruple point $p$, and four tacnodes along a conic at the points $p_{2}, \ldots, p_{5}$. For convenience in the computations to follow, we change this configuration of the branch curve to a sextic and quartic by making a Cremona transformation of the plane.

The Cremona transformation consists of three blow-ups and three blow-downs of $\mathbb{P}^{2}$. First, blow up the plane at $p$ and $p_{1}$ to get exceptional divisors $E$ and $E_{1}$; then blow up the triple point of the branch curve on $E_{1}$ to get an exceptional divisor $F_{1}$.

Next, blow down the proper transform of the line through $p$ and $p_{1}$ to a point on $E \cap E_{1}$, and blow down the proper transform of the line tangent to the octic at $p_{1}$ to a point on $F_{1}$. Finally contract the proper transform of $E_{1}$ to a point on $E \cap F_{1}$, to obtain another $\mathbb{P}^{2}$. Write $\varphi: \mathbb{P}^{2} \rightarrow \mathbb{P}^{2}$ to represent the composition of these maps.

Theorem 1.1. Under this transformation of the plane, the octic $C$ maps to a sextic $S$ with tacnodes at $p_{2}, \ldots, p_{5}$ and one ordinary double point, $q$; the conic $D$ maps to a quartic $Q$ with an ordinary double point at $q$ and a tacnode at a point $q_{1}$ with the tangent direction of $S$, passing through $p_{2}, \ldots, p_{5}$ with tangent direction of $S$.

Proof. Clearly the curves will be unaffected away from the lines $\ell=\overline{p p_{1}}$ and $t$, the tangent line to $C$ at $p_{1}$. Thus $\varphi(C)$ will have tacnodes along $\varphi(D)$ at $p_{2}, \ldots, p_{5}$. The octic meets $\ell$ four times at $p$ and three time at $p_{1}$, so it intersects this line once more. Any point on $\ell$ (other than at $p$ and $p_{1}$ ) is contracted twice: first to a point on $E_{1}$, then again when $E_{1}$ is contracted to $q_{1}$, the point of intersection of $F_{1}$ and $E$. The conic meets $\ell$ twice, away from $p$ and $p_{1}$, so $\varphi(D)$ will have a tacnode at $q_{1}$ with the tangent direction of $\varphi(C)$.

The conic meets the line $t$ twice, away from $p_{1}$, thus $\varphi(D)$ will have a double point at $q$, the contraction of $t$. Since $C$ also meets $t$ twice away from $p_{1}, \varphi(C)$ will also have an ordinary double point.

To see that $\varphi(C)$ is a sextic and $\varphi(D)$ is a quartic, we can count the intersection numbers of each with $E$ or $F_{1}$, as well as compute their self-intersection numbers.

We can realize these three blow-ups and three blow-downs as the quadratic transformation of the plane, $\varphi: \mathbb{P}^{2} \rightarrow \mathbb{P}^{2}$, corresponding to the system of conics through $p$ and $p_{1}$ with a designated tangent direction at $p_{1}$. With coordinates $[x: y: z]$ on $\mathbb{P}^{2}$, where $\ell:(z=0)$ and $t:(x=0)$, this map can be written as $[x: y: z] \rightarrow\left[z^{2}:-x y: x z\right]$.

Thus the configuration of a degree ten curve with the given singularities as an octic and conic is equivalent to a quartic and sextic as above. Under this transformation, the octic and conic given in $[\mathrm{W}]$ become the sextic and quartic defined by the following polynomials. 
$Q:$

$$
\begin{aligned}
& 32 x^{2} y^{2}+23 x^{2} z^{2}-62 x z^{3}+39 z^{4}+\left(-2 x^{2} y z+2 x y z^{2}\right) \alpha \beta \delta \\
& +\left(4 x^{2} z^{2}-10 x z^{3}+6 z^{4}\right) \alpha \beta+\left(-6 x^{2} y z+6 x y z^{2}\right) \beta \delta \\
& +\left(7 x^{2} z^{2}-14 x z^{3}+7 z^{4}\right) \alpha+\left(16 x^{2} z^{2}-38 x z^{3}+22 z^{4}\right) \beta \\
& -\left(16 x^{2} y z-16 x y z^{2}\right) \delta
\end{aligned}
$$

$S:$

$(1.3)$

$$
\begin{aligned}
& 671232 x^{4} y z+2262528 x^{3} y^{3}-1978080 x^{3} y z^{2}-2672640 x^{2} y^{3} z+1981344 x^{2} y z^{3} \\
& +147456 x y^{5}+202752 x y^{3} z^{2}-713376 x y z^{4}+207360 y^{3} z^{3}+38880 y z^{5} \\
& +\left(-96256 x^{4} y^{2}+30369 x^{4} z^{2}+200512 x^{3} y^{2} z-76164 x^{3} z^{3}-46080 x^{2} y^{4}\right. \\
& -99936 x^{2} y^{2} z^{2}+62886 x^{2} z^{4}+18432 x y^{4} z-2304 x y^{2} z^{3}-18756 x z^{5} \\
& \left.+2304 y^{4} z^{2}-2016 y^{2} z^{4}+1665 z^{6}\right) \alpha \beta \delta \\
& -\left(112128 x^{4} y z+377856 x^{3} y^{3}-349824 x^{3} y z^{2}-516096 x^{2} y^{3} z+401280 x^{2} y z^{3}\right. \\
& \left.+165888 x y^{3} z^{2}-201600 x y z^{4}-27648 y^{3} z^{3}+38016 y z^{5}\right) \alpha \beta \\
& +\left(135680 x^{4} y^{2}-43986 x^{4} z^{2}-297344 x^{3} y^{2} z+112488 x^{3} z^{3}+49152 x^{2} y^{4}\right. \\
& +163392 x^{2} y^{2} z^{2}-96780 x^{2} z^{4}+18432 x y^{2} z^{3}+32040 x z^{5}-4608 y^{4} z^{2} \\
& \left.-20160 y^{2} z^{4}-3762 z^{6}\right) \alpha \delta \\
& -\left(396800 x^{4} y^{2}-125193 x^{4} z^{2}-827456 x^{3} y^{2} z+314340 x^{3} z^{3}+187392 x^{2} y^{4}\right. \\
& +421728 x^{2} y^{2} z^{2}-259734 x^{2} z^{4}-73728 x y^{4} z-13824 x y^{2} z^{3}+77220 x z^{5} \\
& \left.+2304 y^{4} z^{2}-4896 y^{2} z^{4}-6633 z^{6}\right) \beta \delta \\
& +\left(162816 x^{4} y z+548352 x^{3} y^{3}-479712 x^{3} y z^{2}-645120 x^{2} y^{3} z+479904 x^{2} y z^{3}\right. \\
& \left.+36864 x y^{3} z^{2}-171936 x y z^{4}+59904 y^{3} z^{3}+8928 y z^{5}\right) \alpha \\
& +\left(462336 x^{4} y z+1557504 x^{3} y^{3}-1441920 x^{3} y z^{2}-2138112 x^{2} y^{3} z\right. \\
& \left.+1654656 x^{2} y z^{3}+681984 x y^{3} z^{2}-832896 x y z^{4}-101376 y^{3} z^{3}+157824 y z^{5}\right) \beta \\
& +\left(559616 x^{4} y^{2}-181410 x^{4} z^{2}-1226624 x^{3} y^{2} z+463656 x^{3} z^{3}+221184 x^{2} y^{4}\right. \\
& +668736 x^{2} y^{2} z^{2}-399084 x^{2} z^{4}-184320 x y^{4} z+78336 x y^{2} z^{3}+132840 x z^{5} \\
& \left.+32256 y^{4} z^{2}-80064 y^{2} z^{4}-16002 z^{6}\right) \delta
\end{aligned}
$$

where $\alpha=\sqrt{17}, \beta=\sqrt{2}$, and $\delta=\sqrt{5+\alpha}$.

Together these curves form a degree ten curve with one ordinary quadruple point, five infinitely near triple points, and no other singularities; this curve is used to construct a numerical Godeaux surface as follows. (See $[\mathrm{W}]$ for details.) Let

$$
\sigma: Y \rightarrow \mathbb{P}^{2}
$$

be the eleven-fold blow-up of the plane resolving these singularities; on $Y$ we have an exceptional curve $E$ with $E=\sigma^{-1}(p)$, five $(-1)$-curves $F_{i}$ and five $(-2)$-curves $E_{i}$, with $E_{i}+F_{i}=\sigma^{-1}\left(p_{i}\right), i=1, \ldots, 5$. The curve $E_{i}$ corresponds to the first blowup of the triple point, with $F_{i}$ the exceptional curve above the intersection of the curve with $E_{i}$.

The proper transforms of the sextic and quartic are smooth and disjoint, and we set the branch curve $B$ of the double cover of $Y$ to be the union of these with $E_{1}, \ldots, E_{5}$. Then $B$ is an even divisor on $Y$ with $B \equiv 2 L$. Let

$$
\pi: X \rightarrow Y
$$

be the double cover; then $K_{X} \equiv \pi^{*}\left(K_{Y}+L\right)$. The points $p, p_{1}, \ldots, p_{5}$ not on a conic imply that $X$ has no global two-forms, thus $p_{g}=0$. Since $X$ is of general type, we then have $q=0$. There are five $E_{i}(-1)$-curves on $X$; contracting these via $\epsilon: X \rightarrow \tilde{X}$ gives a minimal surface $\tilde{X}$ with $K_{\tilde{X}}^{2}=1$. Thus given a sextic and 
quartic as above, we can construct, as a double cover with branch curve the smooth resolution of $Q$ and $S$, a minimal surface of general type with $p_{g}=q=0, K^{2}=1$.

\section{A FOUR-DIMENSIONAL FAMILY OF BRANCH CURVES}

We wish to construct a family of curves with the desired singularities. We start with a smooth cubic curve $C$ and four points $p_{1}, \ldots, p_{4}$ on $C$. Under the group law on the cubic, there are exactly three choices for a point $p_{0}$ on $C$ with $2 \sum_{1}^{4} p_{i}+p_{0}$ non-trivial of order two.

Consider the space of plane sextics with a double point at $p_{0}$ and tacnodes at each $p_{i}$ along $C$; in general there will be a unique sextic satisfying these conditions. The conditions that $2 \sum_{1}^{4} p_{i}+p_{0}$ is not trivial and of order two on $C$, that is

$$
2 \sum_{1}^{4} p_{i}+p_{0} \not \equiv 0 \text { and } 4 \sum_{1}^{4} p_{i}+2 p_{0} \equiv 0,
$$

imply that $C$ is the only cubic through these points and there is a sextic $S$, with the above properties. Thus the linear system of sextics with a double point at $p_{0}$ and tacnodes at each $p_{i}$ (with the tangent direction of $C$ ) is a pencil with generators $S$ and $2 C$.

Next consider quartics with an ordinary double point at $p_{0}$, through each $p_{i}$ with the same tangent direction as that of $C$. The space of quartics through these points is three dimensional. In principle, there are a finite number of these quartics with a tacnode at some point; consider one of these quartics $Q$ with a tacnode at a point $p_{5}$.

There is a unique member of the pencil of sextics through this point $p_{5}$, and it is one condition on this space for the tangent direction of this sextic to coincide with that of the tacnode of $Q$. The sextic and the quartic, if they are irreducible with no other singularities than those mentioned above, together form a branch curve with an order four point and five infinitely near triple points.

We now construct a four-dimensional family of these branch curves. The sextic and quartic given in the previous section, from the transformation of the octic and conic, will give one member of this family.

First fix four distinct points $p_{1}, \ldots, p_{4}$ in the plane. The space of plane cubics is a $\mathbb{P}^{9}$ (since cubics are defined by degree three homogeneous polynomials which have ten coefficients); for a cubic to go through a point puts one condition on this space, thus there is a five-dimensional irreducible space of cubics through $p_{1}, \ldots, p_{4}$.

A smooth plane cubic curve is elliptic, and we can realize it as a torus defined by $\mathbb{C}$ modulo a lattice $\langle 1, \tau\rangle$. Every point on the curve has a representation in the fundamental parallelogram.

By Abel's theorem, the group law on the plane cubic corresponds to the addition in $\mathbb{C}$ modulo $\langle 1, \tau\rangle$. There are three points of order two on the torus, namely $\frac{1}{2}, \frac{\tau}{2}, \frac{1+\tau}{2}$. Thus the projection

$$
\left\{(C, p): 2\left[p+2 \sum_{1}^{4} p_{i}\right] \equiv 0\right\} \rightarrow\left\{C: p_{i} \in C\right\}
$$

is a three-to-one unbranched covering. 
Next, we show that the domain of this map is irreducible. Consider the family $\left(C_{t}, q_{t}\right)$ where $C_{t}=\mathbb{C} \bmod \langle 1, i+t\rangle$,

$$
\begin{aligned}
& p_{1}=0, \quad p_{2}=\frac{1}{2}, \\
& p_{3}=\frac{i}{2}, \quad p_{4}=\frac{i+1}{2},
\end{aligned}
$$

and

$$
q_{t}=\frac{i+t}{2}-2 \sum_{1}^{4} p_{i}=\frac{i}{2}-2 i \bmod \langle 1, i+t\rangle .
$$

Then $q_{t}+2 \sum p_{i}$ is of order two on $C_{t}$. For $t=0, C_{0}$ had lattice $\langle 1, i\rangle$ and $q_{0}=\frac{i}{2}$; for $t=1, C_{1}$ is defined by the lattice $\langle 1, i+1\rangle=\langle 1, i\rangle$ and $q_{1}=\frac{i+1}{2} \not \equiv q_{0}$. Thus we have a continuous path between cubics $\left(C, p_{1}-p_{4}\right)$ with different choices for $p$. In a similar manner we can construct a path such that $q_{0}=\frac{1}{2}$ and $q_{1}=\frac{i+1}{2}$. Thus the fundamental group $\pi_{1}$ acts transitively on the fibers of the map; since the map is finite, the space $\{(C, p)\}$ is irreducible and five-dimensional.

Suppose we are given an element $(C, p)$ of the above space. For a curve to have an ordinary double point at $p$ is three conditions on the space; a tacnode at $p_{i}$ with the tangent direction of $C$ is six conditions, while requiring the curve to go through the point with that tangent direction puts two conditions on the space.

The space of sextics is 27-dimensional, and there are 14 dimensions of plane quartics. There exists a sextic with a double point at $p$ and tacnodes at each $p_{i}$; the conditions

$$
p+2 \sum p_{i} \not \equiv 0, \quad 2 p+4 \sum p_{i} \equiv 0
$$

guarantee that $C$ is the unique cubic determined by these five points and tangent directions of the $p_{i}$, and that there will exist an irreducible sextic $S$ with these singularities. Thus we have a pencil of such curves generated by $S$ and $2 C$. Note that the base points of this pencil are $p, p_{1}, \ldots, p_{4}$, together with the infinitely near points of $p_{1}, \ldots, p_{4}$ corresponding to the tangent directions of $C$.

We also have a three-dimensional system of quartics, each with a double point at $p$, through each $p_{i}$ with the tangent of $C$.

If we choose a general point $p_{5} \in \mathbb{P}^{2}$ (with $(C, p)$ already chosen, and assuming $\left.p_{5} \neq p, p_{1}, \ldots, p_{4}\right)$, it is three conditions on the coefficients for a quartic to have an ordinary double point at $p_{5}$; thus we obtain a unique quartic $Q$ with the above singularities and this additional double point.

Also, there is a unique member of the pencil $\langle S, 2 C\rangle$ through this point. Hence the map

$$
\{(C, p)\} \times \mathbb{P}^{2} \rightarrow\{(Q, S)\}
$$

is birational, and the space of pairs $(Q, S)$ with these singularities is seven dimensional.

Lastly, we look at the subspace of these pairs of curves where $Q$ has a tacnode at $p_{5}$ with the tangent direction of $S$. Since $Q$ already has a double point at $p_{5}$, it is three more conditions on the coefficients of the polynomial defining $Q$ for this curve to have a tacnode. Thus the space of $(Q, S)$, where $Q$ has a tacnode along $S$ at $p_{5}$, is four-dimensional. We have constructed a four-parameter family of reducible branch curves.

Each pair $(Q, S)$ in the family above gives a possible branch curve for the construction of a minimal surface of general type with $p_{g}=q=0, K^{2}=1$. Thus the 
four parameter family of curves gives a four parameter family of numerical Godeaux surfaces.

\section{The Deformation SPACE}

We work with the surface $\tilde{X}$ constructed via the specific equations (1.2) and (1.3). The first cohomology group of the tangent sheaf, $\mathrm{H}^{1}\left(\tilde{X}, \Theta_{\tilde{X}}\right)$, is the tangent space to the deformation space of the surface. The dual to the tangent sheaf $\Theta$ is $\Omega^{1}$, the sheaf of one-forms, thus $h^{i}(\Theta)=h^{2-i}\left(\Omega^{1}(K)\right)$. By Hirzebruch-Riemann-Roch we have

$$
\chi(\Theta)=\chi\left(\Omega^{1}(K)\right)=2 K^{2}-10 \chi .
$$

For the double plane $X, K_{X}^{2}=-4, \chi=1$ and for its minimal model $\tilde{X}, K_{\tilde{X}}^{2}=$ $1, \chi=1$, thus

$$
h^{0}\left(\Theta_{X}\right)-h^{1}\left(\Theta_{X}\right)+h^{2}\left(\Theta_{X}\right)=-18
$$

and

$$
h^{0}\left(\Theta_{\tilde{X}}\right)-h^{1}\left(\Theta_{\tilde{X}}\right)+h^{2}\left(\Theta_{\tilde{X}}\right)=-8 .
$$

Since $X$ and $\tilde{X}$ are of general type, $H^{0}\left(\Theta_{X}\right)=H^{0}\left(\Theta_{\tilde{X}}\right)=0$ (see, for example, $[\mathrm{M}])$. We need to compute $h^{1}\left(\Theta_{\tilde{X}}\right)=8+h^{2}\left(\Theta_{\tilde{X}}\right)=8+h^{0}\left(\Omega_{\tilde{X}}^{1}\left(K_{\tilde{X}}\right)\right)$.

The number of holomorphic tensor fields is a birational invariant, thus

$$
\operatorname{dim} H^{0}\left(\Omega_{\tilde{X}}^{1} \otimes \Omega_{\tilde{X}}^{2}\right)=\operatorname{dim} H^{0}\left(\Omega_{X}^{1} \otimes \Omega_{X}^{2}\right),
$$

and we can compute $h^{2}(\Theta)$ for $X$ in place of its minimal model.

To compute the cohomology of $\Theta_{X}$ and $\Omega_{X}^{1}\left(K_{X}\right)$ on the double plane $X$, we will reduce it to a problem on the rational surface $Y$.

We have the following lemma (see, for example, [C1], Prop. 3.2).

Lemma 3.2. Let $\pi: X \rightarrow Y$ be a double cover with smooth branch locus $B \equiv 2 L$. Then

$$
\pi_{*}\left(\Omega_{X}^{1}\left(K_{X}\right)\right) \cong \Omega_{Y}^{1}\left(K_{Y}+L\right) \oplus\left[\Omega_{Y}^{1}(\log B) \otimes \mathcal{O}_{Y}\left(K_{Y}\right)\right]
$$

The double cover $X$ has a natural involution $\tau$, given locally by $z \rightarrow-z$, where $X$ is $z^{2}=f(x, y)$, and $f=0$ defines $B$ on $Y$. Thus the branch locus is fixed by this involution, and $X / \tau=Y$. This involution also acts naturally on all sheaves on $X$, as well as the cohomology of these sheaves.

In the decomposition of $\Omega_{X}^{1}(K)$, the sheaf $\Omega_{Y}^{1}(\log B) \otimes \mathcal{O}_{Y}\left(K_{Y}\right)$ is the invariant part under the $\mathbb{Z} / 2$-action on the double cover. Similarly, we have the decomposition of $\Theta_{X}$ into its invariant and anti-invariant parts.

Corollary 3.3. We have

$$
\pi_{*}\left(\Theta_{X}\right) \cong \Theta_{Y}(-L) \oplus \Theta_{Y}(-\log B),
$$

where $\Theta_{Y}(-\log B)$ is the $\mathbb{Z} / 2$-invariant component. 
We want to compute $h^{0}\left(\Omega_{X}^{1}\left(K_{X}\right)\right)$; from the lemma, we need to find the global sections of $\Omega_{Y}^{1}\left(K_{Y}+L\right)$ and of $\Omega_{Y}^{1}(\log B) \otimes \mathcal{O}_{Y}\left(K_{Y}\right)$.

We have

$$
\begin{aligned}
K_{Y} & \equiv-3 H+E+\sum\left(E_{i}+2 F_{i}\right), \\
L & \equiv 5 H-2 E-\sum\left(E_{i}+3 F_{i}\right),
\end{aligned}
$$

where $\sigma: Y \rightarrow \mathbb{P}^{2}$ is the resolution of the singularities of the branch curve $B \equiv 2 L$, $H$ is the pullback of the class of a line in the plane, $E_{i}+F_{i}=\sigma^{-1}\left(p_{i}\right), i=1, \ldots, 5$, and $E=\sigma^{-1}\left(p_{0}\right)$.

Proposition 3.4. $\mathrm{H}^{0}\left(\Omega_{Y}^{1}\left(K_{Y}+L\right)\right)=0$.

Proof. Write $K_{Y}+L \equiv 2 H-E-\sum F_{i}$; then $\Omega_{Y}^{1}\left(K_{Y}+L\right)$ is a subsheaf of $\Omega_{Y}^{1}(2 H)$. As $H$ is the pullback of a generic line in $\mathbb{P}^{2}$ which can be chosen away from the singular points of $B$, global sections of $\Omega_{Y}^{1}(2 H)$ are exactly the pullback of global sections of $\Omega_{\mathbb{P}^{2}}^{1}(2)$. By Bott's formula, $h^{0}\left(\Omega_{\mathbb{P}^{2}}^{1}(2)\right)=3$. A basis for this space can be written down as follows.

Choose coordinates $[X: Y: Z]$ on $\mathbb{P}^{2}$ and local charts $\left(x_{i}, y_{i}\right), i=1, \ldots, 3$, with

$$
\begin{aligned}
& x_{1}=\frac{Y}{X}, \quad x_{2}=\frac{X}{Y}, \quad x_{3}=\frac{X}{Z}, \\
& y_{1}=\frac{Z}{X}, \quad y_{2}=\frac{Z}{Y}, \quad y_{3}=\frac{Y}{Z},
\end{aligned}
$$

such that $\left(x_{1}, y_{1}\right)$ is centered at $p$ and $\left(x_{2}, y_{2}\right)$ is centered at $p_{1}$, with the tangent direction of the branch curve at this point given by $x_{2}=0$.

One checks that the three sections

$$
\begin{aligned}
& d x_{1}=\quad-\frac{1}{x_{2}{ }^{2}} d x_{2} \quad=-\frac{y_{3}}{x_{3}{ }^{2}} d x_{3}+\frac{1}{x_{3}} d y_{3}, \\
& d y_{1}=-\frac{y_{2}}{x_{2}{ }^{2}} d x_{2}+\frac{1}{x_{2}} d y_{2}=-\frac{1}{x_{3}{ }^{2}} d x_{3}, \\
& y_{1} d x_{1}-x_{1} d y_{1}=\quad-\frac{1}{x_{2}^{2}} d y_{2} \quad=-\frac{1}{x_{3}^{2}} d y_{3}
\end{aligned}
$$

give a basis for the global sections of $\Omega_{\mathbb{P}^{2}}^{1}(2)$, where we choose $X=0$, or locally $x_{2}=0, x_{3}=0$, to represent the hyperplane class.

Let $\sigma_{0}: Y_{0} \rightarrow \mathbb{P}^{2}$ be the blowup of $p=\left(x_{1}, y_{1}\right)=(0,0)$; write $\left(u_{1}, v_{1}\right)$ and $\left(u_{2}, v_{2}\right)$ for the two charts on $Y_{0}$ above $p$ where $x_{1}=u_{1}, y_{1}=u_{1} v_{1}, x_{1}=u_{2} v_{2}, y_{1}=u_{2}$, and the exceptional curve $E$ is defined by $u_{1}=0, u_{2}=0$. We have $\mathrm{H}^{0}\left(\Omega_{Y_{0}}^{1}(2 H)\right) \cong$ $\mathrm{H}^{0}\left(\Omega_{\mathbb{P}^{2}}^{1}(2 H)\right)$; in fact, if we rewrite the sections using the above transition functions, we obtain a basis for the global sections of $\Omega_{Y_{0}}^{1}(2 H)$, represented in the $\left(u_{1}, v_{1}\right)$ chart by the forms

$$
d u_{1}, v_{1} d u_{1}+u_{1} d v_{1}, u_{1}^{2} d v_{1} .
$$

Since $\mathrm{H}^{0}\left(\Omega_{Y_{0}}^{1}(2 H-E)\right) \subseteq \mathrm{H}^{0}\left(\Omega_{Y_{0}}^{1}(2 H)\right)$, to compute the first space we just find those elements in the span of $d u_{1}, v_{1} d u_{1}+u_{1} d v_{1}, u_{1}^{2} d v_{1}$ which vanish along $E$, which is locally $u_{1}=0$. Thus the only global section is locally given by $u_{1}^{2} d v_{1}$, and $h^{0}\left(\Omega_{Y_{0}}^{1}(2 H-E)\right)=1$.

Next let $\sigma_{1}: Y_{1} \rightarrow Y_{0}$ be the blowup at $p_{1}$, with exceptional divisor $E_{1}$, and let $\sigma_{2}: Y_{2} \rightarrow Y_{1}$ be the blowup of the infinitely near point where $E_{1}$ and the branch curve intersect. By again writing down local charts, we can pull back 
the local section $u_{1}^{2} d v_{1}$ and check that it does not vanish along the exceptional curve $F_{1}$, thus $\mathrm{H}^{0}\left(\Omega_{Y_{2}}^{1}\left(2 H-E-F_{1}\right)\right)=0$, and there are no global sectons of $\Omega_{Y}^{1}\left(2 H-E-\sum F_{i}\right)$.

We must now compute $h^{0}\left(\Omega_{Y}^{1}(\log B) \otimes \mathcal{O}(K)\right)$. Since $\Omega_{Y}^{1}(\log B)$ is a subsheaf of $\Omega_{Y}^{1}(B)$, it suffices to show that there are no global sections of $\Omega_{Y}^{1}\left(K_{Y}+B\right)$. We have

$$
K_{Y}+B \equiv 7 H-3 E-\sum E_{i}-3 \sum F_{i} .
$$

To compute $h^{0}\left(\Omega_{Y}^{1}\left(K_{Y}+B\right)\right)$ we use the method outlined above.

The space $\mathrm{H}^{0}\left(\Omega_{Y}^{1}(7 H)\right)$ is 48 dimensional. First we write down a basis for this space, locally above each of the points $p, p_{1}, \ldots, p_{5}$. A local computation shows that requiring sections to vanish to multiplicity three along $E$ imposes ten conditions, while vanishing four times along $F_{i}$ is nine conditions on the space. To check if there are any forms satisfying these conditions simultaneously, we use the computer algebra package Maple. (Note that this computation is possible, although tedious, to do by hand.) A Maple computation shows that these 55 conditions on the 48-dimensional space have maximal rank, thus there are no global sections and $\mathrm{H}^{0}\left(\Omega_{Y}^{1}\left(K_{Y}+B\right)\right)=0$.

We have therefore shown that

$$
\mathrm{H}^{0}\left(\Omega_{Y}^{1}(\log B) \otimes \mathcal{O}_{Y}\left(K_{Y}\right)\right)=0
$$

and hence have proven the following.

Theorem 3.5. For the minimal surface $\tilde{X}$,

$$
h^{2}\left(\Theta_{\tilde{X}}\right)=0 \quad \text { and } \quad h^{1}\left(\Theta_{\tilde{X}}\right)=8 .
$$

Using the following theorem for the Kuranishi family of deformations (see, for example, $[\mathrm{K}]$ ) we see that the deformation space of $\tilde{X}$ is smooth and complete of dimension eight.

Theorem 3.6. If $\mathrm{H}^{2}\left(\Theta_{\tilde{X}}\right)=0$, then the deformation space is smooth at $\tilde{X}$; if $\mathrm{H}^{0}\left(\Theta_{\tilde{X}}\right)=\mathrm{H}^{2}\left(\Theta_{\tilde{X}}\right)=0$, then the number of moduli is equal to the dimension of $\mathrm{H}^{1}\left(\Theta_{\tilde{X}}\right)$.

Corollary 3.7. The deformation space of $\tilde{X}$ is eight dimensional and smooth at $\tilde{X}$.

\section{The invariant part of the Deformation SPaCe}

Recall the involution of the double cover extends to a $\mathbb{Z} / 2$-action on the minimal model $\tilde{X}$, and on the deformation space. Next we find the subspace of $\mathrm{H}^{1}\left(\Theta_{\tilde{X}}\right)$ which is fixed by the involution.

The invariant part of $\pi_{*} \Omega_{X}^{1}\left(K_{X}\right)$ is $\Omega_{Y}^{1}(\log B) \otimes \mathcal{O}_{Y}\left(K_{Y}\right)$; from the decomposition of the dual sheaf $\Theta_{X}$, we have

$$
\left(\pi_{*} \Theta_{X}\right)^{\mathbb{Z} / 2} \cong \Theta_{Y}(-\log B)
$$

Thus $\mathrm{H}^{1}\left(\Theta_{X}\right)^{\mathbb{Z} / 2} \cong \mathrm{H}^{1}\left(\Theta_{Y}(-\log B)\right) \cong \mathrm{H}^{1}\left(\Omega_{Y}^{1}(\log B) \otimes \mathcal{O}_{Y}\left(K_{Y}\right)\right)$.

From the previous section,

$$
h^{1}\left(\Theta_{X}\right)=h^{1}\left(\Omega_{Y}^{1}\left(K_{Y}+L\right)\right)+h^{1}\left(\Omega_{Y}^{1}(\log B) \otimes \mathcal{O}_{Y}\left(K_{Y}\right)\right)=18 ;
$$

to find $h^{1}\left(\Omega_{Y}^{1}(\log B) \otimes \mathcal{O}_{Y}\left(K_{Y}\right)\right)$ we use the following. 
Proposition 4.1. The sequence

$$
0 \rightarrow \Omega_{Y}^{1} \rightarrow \Omega_{Y}^{1}(\log B) \rightarrow \mathcal{O}_{B} \rightarrow 0
$$

is exact.

Tensoring this sequence with $\mathcal{O}_{Y}\left(K_{Y}\right)$, we obtain

$$
0 \rightarrow \Omega_{Y}^{1}\left(K_{Y}\right) \rightarrow \Omega_{Y}^{1}(\log B)\left(K_{Y}\right) \rightarrow \mathcal{O}_{B}\left(K_{Y}\right) \rightarrow 0
$$

and the corresponding cohomology sequence

$$
0 \rightarrow \mathrm{H}^{0}\left(\mathcal{O}_{B}\left(K_{Y}\right)\right) \rightarrow \mathrm{H}^{1}\left(\Omega_{Y}^{1}\left(K_{Y}\right)\right) \rightarrow \mathrm{H}^{1}\left(\Omega_{Y}^{1}(\log B)\left(K_{Y}\right)\right) \rightarrow 0 .
$$

Riemann Roch gives $h^{1}\left(\Omega_{Y}^{1}\left(K_{Y}\right)\right)=14$. Since $B$ splits as the disjoint union of curves

$$
B=\bigcup_{1}^{5} E_{i} \cup Q \cup S
$$

where each $E_{i}$ is a -2 -curve, $Q$ is rational with $Q^{2}=-4, Q \cdot K=2$, and $S$ is elliptic with $S^{2}=-2, S \cdot K=2$, we obtain $h^{0}\left(\mathcal{O}_{B}\left(K_{Y}\right)\right)=10$.

Thus $h^{1}\left(\Omega_{Y}^{1}(\log B)\left(K_{Y}\right)\right)=4$, and

$$
h^{1}\left(\Theta_{X}\right)^{\mathbb{Z} / 2}=4 \text {. }
$$

We now show that the invariant part of the deformation space of $\tilde{X}$ is four dimensional as well.

We have the exact sequence

$$
0 \rightarrow \Theta_{X}\left(-\log E_{1}, \ldots,-\log E_{5}\right) \rightarrow \Theta_{X} \rightarrow \mathcal{N}_{E_{i}} \rightarrow 0
$$

where the normal bundle of the $E_{i}$ is isomorphic to the direct sum of the sheaves $\mathcal{O}_{E_{i}}\left(E_{i}\right)$. Since each $E_{i}$ is a $(-1)$-curve, this sheaf has trivial cohomology, thus $\mathrm{H}^{1}\left(\Theta_{X}\right) \cong \mathrm{H}^{1}\left(\Theta_{X}\left(-\log E_{1}, \ldots,-\log E_{5}\right)\right)$. By ([C2], Lemma 9.22), this is isomorphic to the first cohomology group of $\Theta_{\tilde{X}} \otimes \mathcal{I}$, where $\mathcal{I}$ is the ideal sheaf of the contracted exceptional curves $E_{i}$ on $\tilde{X}$. Let $Z$ be the set of these five points. We have the sequence

$$
0 \rightarrow \Theta_{\tilde{X}} \otimes \mathcal{I} \rightarrow \Theta_{\tilde{X}} \rightarrow \Theta_{\tilde{X}} \otimes \mathcal{O}_{Z} \rightarrow 0,
$$

and from the resulting sequence of cohomology,

$$
0 \rightarrow \mathrm{H}^{0}\left(\Theta_{\tilde{X}} \otimes \mathcal{O}_{Z}\right) \rightarrow \mathrm{H}^{1}\left(\Theta_{\tilde{X}} \otimes \mathcal{I}\right) \rightarrow \mathrm{H}^{1}\left(\Theta_{\tilde{X}}\right) \rightarrow 0 .
$$

We also obtain the sequence on the invariant parts of each space:

$$
0 \rightarrow \mathrm{H}^{0}\left(\Theta_{\tilde{X}} \otimes \mathcal{O}_{Z}\right)^{\mathbb{Z} / 2} \rightarrow \mathrm{H}^{1}\left(\Theta_{X}\right)^{\mathbb{Z} / 2} \rightarrow \mathrm{H}^{1}\left(\Theta_{\tilde{X}}\right)^{\mathbb{Z} / 2} \rightarrow 0 .
$$

A local computation shows that the global sections of the sheaf $\Theta_{\tilde{X}} \otimes \mathcal{O}_{Z}$ are anti-invariant. Therefore

$$
\mathrm{H}^{1}\left(\Theta_{X}\right)^{\mathbb{Z} / 2} \cong \mathrm{H}^{1}\left(\Theta_{\tilde{X}}\right)^{\mathbb{Z} / 2}
$$

and we have proven the following theorem.

Theorem 4.3. The subspace of the deformation space of $\tilde{X}$ left invariant by the involution is four dimensional. 
In $\S 2$ we constructed a family of branch curves giving a four-dimensional family $\mathcal{X}$ of surfaces, formed as double planes. Now we claim that this family corresponds to the invariant part of the deformation space.

Let $\mathcal{B}$ be the base space of this family, with projection $\omega: \mathcal{X} \rightarrow \mathcal{B}$ and $\omega^{-1}(0)=$ $\tilde{X}$. The $\mathbb{Z} / 2$-action on $\tilde{X}$ extends to a fiber-preserving action on this family of double covers.

Consider the exact sequence

$$
\left.0 \rightarrow \Theta_{\tilde{X}} \rightarrow \Theta_{\mathcal{X}}\right|_{\tilde{X}} \rightarrow \mathcal{N}_{\tilde{X} / \mathcal{X}} \rightarrow 0
$$

and the corresponding long exact sequence of cohomology; the involution acts on all of these sheaves, and trivially on the normal sheaf, since the action preserves fibers.

Taking the $\mathbb{Z} / 2$-invariant part of the cohomology sequence, we obtain the map

$$
\mathrm{H}^{0}\left(\mathcal{N}_{\tilde{X} / \mathcal{X}}\right)^{\mathbb{Z} / 2} \rightarrow \mathrm{H}^{1}\left(\Theta_{\tilde{X}}\right)^{\mathbb{Z} / 2}
$$

The normal sheaf $\mathcal{N}_{\tilde{X} / \mathcal{X}}$ is trivial and invariant under this action, thus

$$
\mathrm{H}^{0}\left(\mathcal{N}_{\tilde{X} / \mathcal{X}}\right)^{\mathbb{Z} / 2}=\mathrm{H}^{0}\left(\mathcal{N}_{\tilde{X} / \mathcal{X}}\right) \cong \mathrm{T}_{0}(\mathcal{B})
$$

and we have the Kodaira-Spencer map for the family $(\mathcal{X}, \mathcal{B})$,

$$
\mathrm{T}_{0}(\mathcal{B}) \rightarrow \mathrm{H}^{1}\left(\Theta_{\tilde{X}}\right)^{\mathbb{Z} / 2} \hookrightarrow \mathrm{H}^{1}\left(\Theta_{\tilde{X}}\right)
$$

Both $\mathcal{B}$ and the invariant part of $\mathrm{H}^{1}\left(\Theta_{\tilde{X}}\right)$ are four-dimensional; we wish to show that the family is smooth at $\tilde{X}$, so that $\operatorname{dim} \mathrm{T}_{0}(\mathcal{B})=\operatorname{dim} \mathcal{B}$, and the family $(\mathcal{X}, \mathcal{B})$ is the invariant component of the deformation space of $\tilde{X}$.

To do so, consider the map from the base space $\mathcal{B}$ to the moduli space $\mathcal{M}$ of the surface $\tilde{X}$,

$$
\mathcal{B} \stackrel{F}{\rightarrow} \mathcal{M}
$$

We first show that no two surfaces in the family $(\mathcal{X}, \mathcal{B})$ are isomorphic, so that the image $F(\mathcal{B})$ is four-dimensional. Consider two surfaces $X_{1}, X_{2} \in \mathcal{X}$; from each we can recover the rational surfaces $Y_{1}, Y_{2}$ and the branch curves $B_{1}, B_{2}$ from which we construct the double covers. If $X_{1}$ and $X_{2}$ are isomorphic, then $Y_{1}=X_{1} / \sigma_{1}$ and $Y_{2}=X_{2} / \sigma_{2}$ must be as well, where $\sigma_{i}$ represents the involution on each double cover. We can then contract the eleven exceptional curves to get the corresponding automorphism of the plane, which will take $B_{1}$ to $B_{2}$.

$$
\begin{aligned}
& X_{1} \cong X_{2} \\
& \stackrel{\downarrow}{\downarrow} \cong \stackrel{\downarrow}{Y_{1}} \cong Y_{2} \\
& \begin{array}{c}
\downarrow \\
\mathbb{P}^{2} \cong \mathbb{P}^{2}
\end{array}
\end{aligned}
$$

To construct the four-parameter family of branch curves, we fix four points $p_{1}, \ldots, p_{4}$ in the plane. The isomorphism $\mathbb{P}^{2} \rightarrow \mathbb{P}^{2}$ above must fix these four points, and therefore it must be the identity. Thus, in order for the two surfaces $X_{1}$ and $X_{2}$ to be isomorphic, the two branch curves $B_{1}, B_{2}$ must be identical. 
Thus the image of this family in the moduli space of $\tilde{X}, F(\mathcal{B}) \subseteq \mathcal{M}$, is fourdimensional. Since each member of $F(\mathcal{B})$ is a double cover, this image is in the invariant part of the moduli space, and we have

$$
T_{\tilde{X}}(F(\mathcal{B})) \subseteq \mathrm{H}^{1}\left(\Theta_{\tilde{X}}\right)^{\mathbb{Z} / 2} .
$$

Since $F(\mathcal{B})$ is four-dimensional, the tangent space is at least four-dimensional. From Theorem 4.3 , the $\mathbb{Z} / 2$-invariant part of $\mathrm{H}^{1}\left(\Theta_{\tilde{X}}\right)$ is four-dimensional, thus we must have

$$
T_{\tilde{X}}(F(\mathcal{B}))=\mathrm{H}^{1}\left(\Theta_{\tilde{X}}\right)^{\mathbb{Z} / 2} .
$$

Hence the dimension of the tangent space of $F(\mathcal{B})$ at $\tilde{X}$ is equal to the dimension of $F(\mathcal{B})$, and we conclude that this space is smooth at $\tilde{X}$.

\section{REFERENCES}

[Ba] Barlow, R., Some new surfaces with $p_{g}=0$, Duke Math. J. 51 (1984), 889-904. MR 86c: 14032

[B-P-V] Barth, W., Peters, C., Van de Ven, A., Compact Complex Surfaces, Ergebnisse der Mathematik und ihrer Grenzgebiete (3 Folge), vol. 4, Berlin-Heidelberg-New York: Springer. 1984. MR 86c:32026

[Bo] Bott, R., Homogeneous vector bundles, Annals of Math. 66 (1957), 203-248. MR 19:681d

[Ca] Campedelli, L., Sopra alcuni piani doppi notevoli con curva di diramazioni del decimo ordine, Atti Acad. Naz. Lincei 15 (1931), 536 - 542.

[C1] Catanese, F., On the moduli spaces of surfaces of general type, J. Differential Geometry 19 (1984), 483-515. MR 86h:14031

[C2] Catanese, F., Moduli of Algebraic Surfaces, Lecture Notes in Mathematics 1337 (1985). Theory of Moduli, C.I.M.E. MR 89i:14031

[H] Hartshorne, R., Algebraic Geometry, New York-Heidelberg-Berlin: Springer. 1977. MR $\mathbf{5 7 : 3 1 1 6}$

[I] Inoue, M., Some new surfaces of general type, Tokyo J. Math. 17 no. 2 (1994), 295-319. MR 95j: 14048

[K] Kodaira, K., Complex Manifolds and Deformations of Complex Structures, New York: Springer-Verlag. 1986. MR 87d:32040

[M] Matsumura, H., On algebraic groups of birational transformations, Rend. Accad. Lincei Ser. (8) 34 (1963), 151-155. MR 29:5819

[O-P] Oort, F., Peters, C., A Campedelli surface with torsion group $\mathbb{Z} / 2$, Nederl. Akad. Wetensch. Indag. Math. 43 (1981), 399-407. MR 83h:14032

[W] Werner, C., A surface of general type with $p_{g}=q=0, K^{2}=1$, Manuscripta Math. 84 (1994), 327-341. MR 95g:14042

Department of Mathematics, University of Michigan, Ann Arbor, Michigan 48109

E-mail address: cwerner@math.1sa.umich.edu 